\title{
Evaluación a nivel laboratorio de la capacidad de remoción de materia orgánica de Chlorella vulgaris en las aguas residuales de la PTAR Salitre
}

\author{
Laboratory evaluation of the organic matter removal capacity of Chlorella vulgaris in \\ wastewater from the Salitre WWTP
}

\author{
Juan Andrés Sandoval Herrera \\ Ingeniero Químico de la Universidad Industrial de Santander \\ Profesor de la Universidad de América, Bogotá, Colombia \\ https://orcid.org/0000-0001-8957-1421 \\ juan.sandoval@profesores.uamerica.edu.co
}

Brian Orlando Malo Malo Ingeniero Químico de la Universidad de América, Bogotá, Colombia

Julián Camilo Cartagena Arévalo

Ingeniero Químico de la Universidad de América, Bogotá, Colombia

Diego Rubio Fernández

Biólogo de la Universidad Militar Nueva Granada, Maestría en Ciencias de la Universidad Nacional de Colombia sede Bogotá, Colombia Profesor de la Universidad de América, Bogotá, Colombia https://orcid.org/0000-0003-0760-9567

diego.rubio@investigadores.uamerica.edu.co

Fecha de recepción: 15 de septiembre del 2017

Fecha de aceptación: 2 de julio del 2018

\begin{abstract}
Sugerencia de citación: Sandoval Herrera, J. A., Malo Malo, B. O., Cartagena Arévalo, J.C. y Rubio Fernández, D. (2018). Evaluación a nivel laboratorio de la capacidad de remoción de materia orgánica de Chlorella vulgaris en las aguas residuales de la PTAR Salitre. Mutis, 8(1), 34-42, doi: http://dx.doi.org/10.21789/22561498.1368
\end{abstract}

Editor: Gerard Olivar-Tost

\section{RESUMEN}

El proceso primario realizado por la Planta de Tratamiento de Aguas Residuales (PTAR) Salitre no logra una remoción mayor del $60 \%$ de materia orgánica. En esta investigación se realizó un biotratamiento con la microalga Chlorella vulgaris de las aguas residuales tratadas por esta planta, antes de su vertimiento, haciendo énfasis en la remoción de materia orgánica por demanda química de oxígeno y sólidos suspendidos totales, para evaluar su eficiencia. También se evaluó la incidencia de la microalga en otros parámetros fisicoquímicos. Una vez culminado el biotratamiento, se analizaron los parámetros mencionados en el sobrenadante para comparar los resultados del tratamiento químico realizado por la PTAR Salitre (remoción por DQO de 35,30\% y 57,51\% por SST) con el mejor resultado del biotratamiento (muestra 1: $81,80 \%$ por DQO y $89,91 \%$ por SST). Finalmente, se obtuvo un mejor porcentaje de remoción de DQO con respecto a los resultados de trabajos similares de otros autores.

Palabras clave: biorremediación, aguas residuales, remoción de materia orgánica, microalga. 


\begin{abstract}
The primary process carried out by the Salitre Wastewater Treatment Plant (WWTP) does not achieve a removal rate higher than $60 \%$ of organic matter. In this research, a biotreatment was done with the microalgae Chlorella vulgaris of the wastewater treated by this plant, prior to its dumping, with an emphasis on the removal of organic matter by chemical demand of oxygen and total suspended solids, in order to evaluate its efficiency. The incidence of microalgae in other physicochemical parameters was also evaluated. At the end of the biotreatment, the mentioned parameters were analyzed in the supernatant to compare the results of the chemical treatment performed by the Salitre WWTP (COD removal of $35.30 \%$ and $57.51 \%$ by SST) with the best biotreatment result (Sample $1: 81.80 \%$ by COD and $89.91 \%$ by SST). Finally, a better CDO removal percentage was achieved in relation to similar works by other authors.
\end{abstract}

Keywords: bioremediation, wastewater, organic matter removal, microalgae.

\section{INTRODUCCIÓN}

La Planta de Tratamiento de Aguas Residuales (PTAR) Salitre, ubicada en el noroccidente de la ciudad de Bogotá, trata $4 \mathrm{~m}^{3} / \mathrm{s}$ de aguas residuales provenientes de la cuenca El Salitre-Torca, la cual está conformada por seis localidades: Suba, Engativá, Chapinero, Barrios Unidos, Teusaquillo y Usaquén, y tiene una población de 2.850.200 personas. El tratamiento que se realiza en esta planta es de tipo primario químicamente asistido (Guerrero, 1996; Metcalf \& Eddy Inc., 1981; Ramalho, 2003; Romero, 2004).

Aunque por ahora no se realiza ningún otro tratamiento en la PTAR Salitre, hoy en día existen nuevas tecnologías para el tratamiento de aguas residuales domiciliarias como la electrocoagulación, la radiación ultravioleta, el ultrasonido y la biorremediación. Esta última consiste en la utilización de microorganismos para depurar contaminantes en el suelo y el agua (Acosta, 2004; Chacón, Andrade, Cárdenas, Araujo y Morales, 2004; Garbisu, Amézaga y Alkorta, 2002; Tafur y Estrada, 2015); por ejemplo, la biorremediación emplea microalgas como la Chlorella vulgaris (Herrera, 2010; Olarte y Valencia, 2016; Wang et al., 2010). No obstante, esta tecnología aún no está implementada a gran escala en Colombia.

El objetivo del presente artículo es evaluar el uso de la microalga Chlorella vulgaris para la remoción de materia orgánica de las aguas tratadas en la PTAR Salitre a nivel de laboratorio, mediante la adecuación de las condiciones de cultivo que garanticen su crecimiento; el acondicionamiento de la microalga al nuevo sustrato: agua residual; y el análisis de los parámetros fisicoquímicos relacionados con la eventual disminución de carga de materia orgánica en el efluente.

\section{MATERIALES Y MÉTODOS}

\section{Características del agua residual}

El primer paso del tratamiento realizado en la PTAR Salitre consiste en la retención de los residuos sólidos en el cribado grueso, mediante rejas paralelas separadas entre sí cinco centímetros. De ahí, el agua es conducida al cribado fino, donde los residuos pequeños son retirados por medio de rastrillos $y$ depositados en una banda transportadora que los lleva, junto con los residuos gruesos removidos en el cribado grueso, hacia el contenedor para su disposición final. A continuación, en los canales de grasas y desarenadores, se adiciona cloruro férrico y un polielectrolito aniónico al agua para facilitar la coagulación de las partículas en suspensión y separar las arenas y grasas por sedimentación y decantación, respectivamente. Después, el agua pasa a unos decantadores para remover la materia que aún contiene. Finalmente, se vierte al río Bogotá con un porcentaje de $40 \%$ a $50 \%$ de materia orgánica (Empresa de Acueducto, Alcantarillado y Aseo de Bogotá, s.f.).

El punto de partida de este proyecto fue el análisis de las propiedades fisicoquímicas del agua que sale de la planta para ser vertida al río Bogotá. En la Tabla 1 se presentan los valores promedio del muestreo puntual, realizado el 5 de septiembre de 2016. 
Tabla 1. Caracterización fisicoquímica del agua residual tratada

\begin{tabular}{ccc}
\hline Característica fisicoquímica & Resultado & Unidad \\
\hline $\mathrm{pH}$ & 7,63 & \\
Alcalinidad & 225 & $\mathrm{mg}-\mathrm{CaCO}_{3} / \mathrm{L}$ \\
Conductividad & 833 & $\mu \mathrm{s} / \mathrm{cm}$ \\
Sólidos suspendidos totales (SST) & 116 & $\mathrm{mg} / \mathrm{L}$ \\
Sólidos totales (ST) & 704 & $\mathrm{mg} / \mathrm{L}$ \\
Sólidos suspendidos volátiles (SSV) & 92 & $\mathrm{mg} / \mathrm{L}$ \\
Sólidos volátiles (SV) & 410 & $\mathrm{mg} / \mathrm{L}$ \\
DQO & 361 & $\mathrm{mg} / \mathrm{L}-\mathrm{O}_{2}$ \\
Turbiedad & 89 & $\mathrm{NTU}$ \\
\hline
\end{tabular}

Fuente: elaboración propia con base en datos de la PTAR Salitre del 5 de septiembre del 2016.

\section{Desarrollo del cultivo}

La finalidad de esta etapa del proceso fue obtener una cantidad suficiente de biomasa de microalga Chlorella vulgaris para llevar a cabo las pruebas posteriores.

Condiciones iniciales. Con base en la literatura consultada (Abalde, Cid, Fidalgo, Torres y Herrero, 1995; Ángel y Pimienta, 2012; Monroy, 2014; Moronta, Mora y Morales, 2006; Pearsall y Loose, 1936; Velasco, Barros-Gómez, Ospina-Salazar y Trujillo, 2009), se establecieron las condiciones iniciales: agitación del cultivo por medio de flujo de aire continuo a $4 \mathrm{~L} / \mathrm{min}$; iluminación mediante luz natural; fotoperiodo de 12 horas de luz por 12 horas de oscuridad; pH entre 6 y 7 durante todo el cultivo; temperatura entre 17 y $25^{\circ} \mathrm{C}$; se emplearon cuatro recipientes de vidrio de $250 \mathrm{~mL}$, a los que se adicionaron $200 \mathrm{~mL}$ de medio de cultivo, compuesto por agua y fertilizante agrícola de nombre comercial Foliagro, y $20 \mathrm{~mL}$ de microalga; tanto los materiales como el medio de cultivo Foliagro fueron previamente esterilizados mediante autoclave a 121 ${ }^{\circ} \mathrm{C}$ y 15 psi durante 30 minutos.
Escalamiento. A los 16 días, después de evaluar que el crecimiento de la microalga había alcanzado su fase estacionaria, se realizó un primer escalamiento a dos recipientes de $4 \mathrm{~L}$, manteniendo la misma concentración de microalga. En cada recipiente se agregaron $1 \mathrm{~L}$ de medio de cultivo y $100 \mathrm{~mL}$ de microalga cultivada en los recipientes iniciales. A los 33 y a los 41 días se repitió el escalamiento, con las mismas cantidades de medio de cultivo y de microalga, hasta obtener 3,3 L de cultivo en cada recipiente.

\section{Preexperimentación}

Cabe aclarar que, en este estudio, se utilizó agua residual tratada (ART) sin esterilizar, que no fue objeto de ensayos microbiológicos debido a las limitaciones del estudio. Por lo tanto, fue necesario verificar la capacidad de adaptación de la microalga a este nuevo sustrato. Para esto, se prepararon cuatro muestras cuya composición se indica en la Tabla 2. El volumen de cultivo restante se empleó para evaluar la incidencia de la adaptación previa de la microalga en los resultados de la remoción de materia orgánica.

Tabla 2. Composiciones de los cultivos de microalga usados en la preexperimentación

\begin{tabular}{ccc}
\hline Muestra & Volumen & $\begin{array}{c}\text { Porcentaje de vol. de } \\
\text { microalga }\end{array}$ \\
\hline Muestra 1 & $150 \mathrm{~mL}$ microalga /150 mL ART & $50 \%$ \\
Muestra 2 & $90 \mathrm{~mL}$ microalga / 210 mL ART & $30 \%$ \\
Muestra 3 & $30 \mathrm{~mL}$ microalga / 270 mL ART & $10 \%$ \\
\hline
\end{tabular}

Fuente: elaboración propia. 


\section{Desarrollo de la biorremediación en el laboratorio}

Las condiciones empleadas en esta etapa del proceso fueron las mismas que se usaron en la preexperimentación. En la Tabla 3 se indica la composición de las muestras preparadas. Las muestras preparadas con microalga adaptada (MA) son la 4, la 5 y la 6; mientras que las preparadas con microalga no adaptada (MNA) son la 7, la 8 y la 9.

Tabla 3. Composiciones de los cultivos de microalga usados en la experimentación

\begin{tabular}{ccc}
\hline Muestra & Volumen & $\begin{array}{c}\text { Porcentaje de vol. de } \\
\text { microalga }\end{array}$ \\
\hline Muestra 4 & $100 \mathrm{~mL} \mathrm{MA} \mathrm{/} \mathrm{900} \mathrm{mL} \mathrm{ART}$ & $10 \%$ \\
Muestra 5 & $200 \mathrm{~mL} \mathrm{MA} \mathrm{/} \mathrm{800} \mathrm{mL} \mathrm{ART}$ & $20 \%$ \\
Muestra 6 & $300 \mathrm{~mL} \mathrm{MA} \mathrm{/} \mathrm{700} \mathrm{mL} \mathrm{ART}$ & $30 \%$ \\
Muestra 7 & $100 \mathrm{~mL} \mathrm{MNA} \mathrm{/} \mathrm{900} \mathrm{mL} \mathrm{ART}$ & $10 \%$ \\
Muestra 8 & $200 \mathrm{~mL} \mathrm{MNA} \mathrm{/} \mathrm{800} \mathrm{mL} \mathrm{ART}$ & $20 \%$ \\
Muestra 9 & $300 \mathrm{~mL} \mathrm{MAN} \mathrm{/} \mathrm{700} \mathrm{mL} \mathrm{ART}$ & $30 \%$ \\
Blanco & $1000 \mathrm{~mL} \mathrm{ART}$ & $0 \%$ \\
\hline
\end{tabular}

Fuente: elaboración propia.

\section{Análisis}

Crecimiento del cultivo. La concentración celular se midió por medio del conteo de células en la cámara de Neubauer (Arredondo y Voltolina, 2007; Bastidas, s.f.; Universidad del País Vasco, s.f. ). La velocidad de crecimiento por día $(\mu)$ se calculó de la siguiente manera según Wang et al. (2010):

$$
\mu=\frac{\ln \left(\frac{X_{2}}{X_{1}}\right)}{t_{2}-t_{1}}
$$

Donde $X_{1}$ y $X_{2}$ son la concentración celular inicial y final, respectivamente, $y$ es el tiempo en días.

Parámetros fisicoquímicos. Después de los 15 días del biotratamiento, en la etapa final, se separó la microalga de su medio de cultivo, empleando centrifugación (Muylaert, K., et al, 2018). La biomasa residual fue dispuesta como abono orgánico. El líquido sobrenadante se empleó en los análisis fisicoquímicos realizados de acuerdo con los estándares internacionales (American Public Health Association [APHA], American Water Works Association [AWWA], Water Environment Federation [WEF], 2012): sólidos totales, sólidos volátiles, sólidos suspendidos volátiles, pH, turbiedad (Carpio, 2007), alcalinidad y conductividad (Recinto Universitario de Mayagüez, s.f.). El porcentaje de remoción de materia orgánica se determinó con base en la variación de dos parámetros: sólidos suspendidos totales (SST) y demanda química de oxígeno (DQO), según los procedimientos sugeridos por APHA et al. (2012). La ecuación que se utilizó en ambos casos fue:

$$
\% R=\frac{\text { Entrada }(\text { agua cruda })-\text { Salida }(\text { agua tratada })}{\text { Entrada }(\text { a.gua } \text { cruda })} \times 100 \%
$$

Donde $\% R$ es el porcentaje de remoción; Entrada es el valor inicial del parámetro (SST o DQO) en el agua cruda que, en este caso, es el agua residual tratada por la PTAR; y Salida es el valor final después del tratamiento.

\section{RESULTADOS Y DISCUSIÓN}

\section{Crecimiento del cultivo}

Preexperimentos. En la Tabla 4 se presentan los resultados promedio de los conteos realizados para las muestras con microalga no adaptada al ART en los diferentes porcentajes volumétricos empleados. 
Tabla 4. Velocidad de crecimiento específico en los preexperimentos

\begin{tabular}{cccccc}
\hline Muestra & $\begin{array}{c}\text { Porcentaje de vol. de } \\
\text { microalga }\end{array}$ & $\begin{array}{c}\text { Concentración celular } \\
\text { inicial } \\
(\text { cél/mL) }\end{array}$ & $\begin{array}{c}\text { Concentración celular } \\
\text { final } \\
(\text { cél/mL) }\end{array}$ & Tiempo (días) & $\begin{array}{c}\text { Tasa de crecimiento } \\
\left(\text { cél } \mathbf{m L}^{-1} \mathbf{d}^{-1}\right)\end{array}$ \\
\hline 1 & $50 \%$ & $1 \mathrm{E}+07$ & $1,2 \mathrm{E}+07$ & 11 & 0,018 \\
2 & $30 \%$ & $2,9 \mathrm{E}+06$ & $7,6 \mathrm{E}+06$ & 15 & 0,069 \\
3 & $10 \%$ & $2,4 \mathrm{E}+06$ & $4,7 \mathrm{E}+06$ & 15 & 0,048 \\
\hline
\end{tabular}

Fuente: elaboración propia.

Como se observa en la tabla anterior, la mayor velocidad específica de crecimiento se obtuvo con un $30 \%$ de volumen de microalga y la menor, con un $50 \%$; esta última evidenció el inicio de la fase de muerte a los 11 días, por lo que se detuvo el cultivo en ese tiempo. Se podría decir que el crecimiento óptimo de la microalga para este nuevo sustrato (ART) se encontraría entre 10 y $30 \%$ de volumen. Otros autores han encontrado 0,109 cél $\mathrm{mL}^{-1} \mathrm{~d}^{-1}$ para Chlorella sp. en cultivos in vitro con agua residual de la ciénaga Miramar (Barrancabermeja, Santander) (Tafur y Estrada, 2015), 0,42 cél $\mathrm{mL}^{-1} \mathrm{~d}^{-1}$ para Synechocystis minuscula en cultivos con agua residual urbana (Jonte, Rosales, Yépez, Beltrán y Morales, 2007) y una velocidad de crecimiento específica de 0,296 $\mathrm{d}^{-1}$ para Chlorella sp. en cultivo a nivel de laboratorio con purines (Cervera, 2011). El menor crecimiento obtenido en este estudio se debe posiblemente a la no esterilización del ART.

Fase de biotratamiento. En la Tabla 5 se muestran los resultados de la evaluación del crecimiento celular en las muestras 4 a 9.

Tabla 5. Velocidad de crecimiento específico en los preexperimentos

\begin{tabular}{|c|c|c|c|c|}
\hline Muestra & $\begin{array}{l}\text { Porcentaje de vol. } \\
\text { de microalga }\end{array}$ & $\begin{array}{c}\text { Concentración } \\
\text { celular inicial } \\
(\text { cél } / \mathrm{mL})\end{array}$ & $\begin{array}{c}\text { Concentración } \\
\text { celular final } \\
\text { (cél/mL) }\end{array}$ & $\begin{array}{l}\text { Tasa de crecimiento } \\
\text { (cél } \mathrm{mL}^{-1} \mathrm{~d}^{-1} \text { ) }\end{array}$ \\
\hline 4 & $10 \% \mathrm{MA}$ & $1,4 \mathrm{E}+06$ & $3 E+06$ & 0,054 \\
\hline 5 & $20 \% \mathrm{MA}$ & $2,5 \mathrm{E}+06$ & $3,7 E+06$ & 0,028 \\
\hline 6 & $30 \% \mathrm{MA}$ & $2,3 E+06$ & $4,7 \mathrm{E}+06$ & 0,051 \\
\hline 7 & $10 \% \mathrm{MNA}$ & $1,1 \mathrm{E}+06$ & $3,7 E+06$ & 0,087 \\
\hline 8 & $20 \%$ MNA & $2,4 E+06$ & $4,3 \mathrm{E}+06$ & 0,042 \\
\hline 9 & $10 \%$ MNA & $2,4 \mathrm{E}+06$ & $5,1 \mathrm{E}+06$ & 0,054 \\
\hline
\end{tabular}

Fuente: elaboración propia.

Como se observa en la tabla anterior, la muestra 7 obtuvo la mayor velocidad de crecimiento (10\% MNA) con 0,087 cél $\mathrm{mL}^{-1} \mathrm{~d}^{-1}$. Las muestras $4(10 \% \mathrm{MA})$ y 9 (30\% MNA) también presentaron un alto valor. La muestra 2 obtuvo el menor crecimiento (20\% MA) con 0,028 cél $\mathrm{mL}^{-1} \mathrm{~d}^{-1}$. Por tanto, a igual concentración volumétrica, las muestras no adaptadas al ART alcanzan la mayor velocidad de crecimiento específico. Finalmente, es claro que, en promedio, las mayores tasas de crecimiento celular se alcanzan con $10 \%$ de microalga, adaptada o no.

\section{Remoción de materia orgánica}

En la PTAR. Aunque la PTAR Salitre no realiza un tratamiento biológico secundario, los resultados de remoción de materia orgánica se toman como línea base para futuras implementaciones de nuevas 
tecnologías. Para tal efecto, el día 5 de septiembre de 2016 se obtuvieron los siguientes resultados de remoción de materia orgánica según registros de la PTAR Salitre:

Porcentaje de remoción por sólidos suspendidos totales (SST):

$$
\% R_{S S T}=\frac{273^{m g} / L-116^{m g} / L}{273 m g / L} x 100 \%=57,51 \%
$$

Porcentaje de remoción por demanda química de oxígeno (DQO):
$\% R_{D Q O}=\frac{558 \frac{\mathrm{mg}}{\mathrm{LO}}-361 \mathrm{mg} / \mathrm{L} \mathrm{O}_{2}}{558 \mathrm{mg} / \mathrm{L} \mathrm{O}_{2}} \times 100 \%=35,30 \%$

Con la microalga Chlorella vulgaris. Tras finalizar el biotratamiento y separar la biomasa de su medio de cultivo, se realizó el análisis de los parámetros fisicoquímicos del líquido sobrenadante y se obtuvieron los porcentajes que se muestran en la Tabla 6.

Tabla 6. Porcentaje de remoción de materia orgánica

\begin{tabular}{ccc}
\hline Muestra & Porcentaje de remoción de SST & $\begin{array}{c}\text { Porcentaje de } \\
\text { remoción de DQO }\end{array}$ \\
\hline 4 & 89,91 & 81,80 \\
5 & 79,31 & 73,19 \\
6 & 72,84 & 73,43 \\
8 & 68,19 & 71,83 \\
9 & 55,17 & 64,99 \\
Blanco & 57,76 & 64,99 \\
\hline
\end{tabular}

Fuente: elaboración propia.

Como se indica en la tabla anterior, la muestra 4, correspondiente al $10 \%$ de volumen de microalga previamente adaptada al agua residual, fue la que presentó mejores porcentajes de remoción. El blanco presentó resultados similares a los alcanzados por dicha muestra.
En la Tabla 7 se resumen los valores de los parámetros fisicoquímicos obtenidos en la muestra que reportó mejores resultados (muestra 4), el blanco analítico y la caracterización del agua residual tratada por la PTAR Salitre (ART) con la que se inició el estudio.

Tabla 7. Resultados de los parámetros fisicoquímicos

\begin{tabular}{cccc}
\hline Parámetro & \multicolumn{3}{c}{ Resultados } \\
\cline { 2 - 4 } & ART & Blanco & Muestra 4 \\
\hline Sólidos totales $(\mathrm{mg} / \mathrm{L})$ & 704 & 367 & 364 \\
Sólidos volátiles $(\mathrm{mg} / \mathrm{L})$ & 410 & 119 & 99 \\
Sólidos suspendidos volátiles $(\mathrm{mg} / \mathrm{L})$ & 92 & 18 & 13 \\
Turbiedad $(\mathrm{NTU})$ & 7,63 & 8,08 & 6,3 \\
Alcalinidad $(\mathrm{mg}-\mathrm{CaCO} / \mathrm{L})$ & 89 & 11 & 7 \\
Conductividad $(\mu \mathrm{s} / \mathrm{cm})$ & 225 & 134 & 19 \\
Sólidos suspendidos totales $(\mathrm{mg} / \mathrm{L})$ & 833 & 985 & 609 \\
Demanda química de oxígeno $\left(\mathrm{mg} / \mathrm{L}-\mathrm{O}_{2}\right.$ ) & 116 & 20 & 13 \\
& 361 & 78 & 73 \\
\hline
\end{tabular}

Fuente: elaboración propia. 
Aunque los valores que se obtuvieron en el blanco son similares a los de la mejor muestra de microalga (10\% de volumen), la remoción que alcanzó esta última es mayor, particularmente en cuanto a DQO. Finalmente, en la Tabla 8 se comparan los resultados obtenidos en el presente estudio con aquellos obtenidos por otros autores; se encontró que solamente un estudio (Zhou et al., 2012) reportó un porcentaje mayor de remoción de DQO pero con una especie de microalga diferente.

Tabla 8. Comparación con resultados de otros estudios

\begin{tabular}{|c|c|c|c|}
\hline I & $\% R_{\text {DQO }}$ & Especie & Referencia \\
\hline $0,412 d-1$ & 50,9 & Chlorella sp. & Wang et al. (2010) \\
\hline $1,510 \mathrm{gL}-1 \mathrm{~d}-1$ & 89,0 & $\begin{array}{c}\text { Auxenochlorella } \\
\text { protothecoides }\end{array}$ & Zhou et al. (2012) \\
\hline $0,200 \mathrm{gL}-1 \mathrm{~d}-1$ & 77,0 & Chlorella kessleri & Li et al. (2012) \\
\hline $0,125 \mathrm{gL}-1 \mathrm{~d}-1$ & 70,0 & Chlorella protothecoide & Li et al. (2012) \\
\hline 0,284 cél mL-1d-1 & 76,0 & Chlorella vulgaris & Herrera (2010) \\
\hline No disponible & 54,8 & Chlorella sp. & Chacón et al. (2004) \\
\hline 0,176 cél mL-1d-1 & 35,6 & Scenedesmus sp. & Andrade, Vera, Cárdenas y Morales (2009) \\
\hline 0,086 gL-1d-1 & 30,9 & Chlorella vulgaris & Olarte y Valencia (2016) \\
\hline 0,054 cél mL-1d-1 & 81,8 & Chlorella vulgaris & Este estudio \\
\hline
\end{tabular}

Fuente: elaboración propia.

\section{CONCLUSIONES}

Se logró adecuar el cultivo de la microalga Chlorella vulgaris empleando agua residual como parte de su medio de cultivo. Las muestras cultivadas con microalga adaptada presentaron mejores resultados en cuanto a remoción de materia orgánica que las no adaptadas. Los crecimientos alcanzados son bajos en comparación con lo que se reporta en la literatura, con diferentes tipos de aguas residuales industriales y domésticas; sin embargo, los porcentajes de remoción de materia orgánica por DQO obtenidos en este estudio son mayores, excepto en un caso reportado (ver Tabla 8). La muestra 4 (MA con 10\% de volumen de microalga) alcanzó porcentajes de remoción de materia orgánica por DQO de $81,80 \%$ y por SST de $89,91 \%$, los cuales son mayores que aquellos registrados en la PTAR Salitre con tratamiento primario, donde los valores fueron de $35,30 \%$ por DQO y $57,51 \%$ por SST.

Se encontró que no existe una relación directa entre la velocidad de crecimiento del cultivo y la remoción de materia orgánica: la muestra 1 obtuvo mejores resultados en los análisis fisicoquímicos realizados después del biotratamiento aun teniendo una de las menores velocidades de crecimiento $\left(0,028\right.$ cél $\left.\mathrm{mL}^{-1} \mathrm{~d}^{-1}\right)$, lo cual confirma las observaciones realizadas por Wang et al. (2010). El blanco analítico reportó resultados muy similares a los de la muestra 1 posiblemente debido a que, al utilizar inyección de aire como fuente de agitación en el laboratorio, el oxígeno realiza un tratamiento biológico sobre el agua residual, lo cual no justificaría el uso de la microalga para la remoción de materia orgánica a escala de laboratorio; aunque a escala industrial la inyección de oxígeno no es rentable económicamente, la agitación se lograría por medio del flujo continuo del agua residual. Para futuras investigaciones, se sugiere tener en cuenta la concentración de oxígeno disuelto a fin de entender el mecanismo de reducción de materia orgánica desarrollado por la microalga, así como esterilizar el agua residual para eliminar cualquier microoganismo competidor. 


\section{REFERENCIAS}

Abalde, J., Cid, A., Fidalgo, P., Torres, E. y Herrero, C. (1995). Microalgas: cultivos y aplicaciones. Monografía N.o 26. La Coruña, España: Universidade da Coruña.

Acosta, E. (2004). Evaluación de la remoción de nutrientes de aguas de porqueriza mediante el uso de microalgas. Colombia: Universidad Nacional de Colombia.

American Public Health Association, American Water Works Association, Water Environment Federation. (2012). Standard Methods for the examination of water and wastewater.

Andrade, C., Vera, A., Cárdenas, C. y Morales, E. (2009). Biomass production of microalga Scenedesmus sp. with wastewater from fishery. Revista Técnica de la Facultad de Ingeniería. Universidad del Zulia, 32(2), 126-134.

Ángel, C. y Pimenta, W. (2012). Evaluación de la incidencia del nitrógeno en el medio de cultivo bold basal para la producción de biomasa de la microalga Chlorella vulgaris en un fotobiorreactor, para la obtención de ácidos grasos. Colombia: Fundación Universidad de América.

Arredondo, B. y Voltolina, D. (2007). Métodos y herramientas analíticas en la evaluación de la biomasa microalgal. Centro de Investigaciones Biológicas del Noroeste.

Bastidas, O. (s.f.). Conteo celular con hematocitómetro. Uso elemental del hematocitómetro. Recuperado de http://www.celeromics.com/es/resources/ docs/Articles/Conteo-Camara-Neubauer.pdf

Carpio, T. (2007). Turbiedad por nefelometría (método B). Bogotá, Colombia: Instituto de Hidrología, Meteorología y Estudios Ambientales.

Cervera del Castillo, O. (2011). Tratamiento de purines para la producción de biomasa microalgal. (Tesis de maestría). Universidad de Almería, España.

Chacón, C., Andrade, C., Cárdenas, C., Araujo, I. y Morales, E. (2004). Uso de Chlorella sp. y Scenedesmus sp. en la remoción de nitrógeno, fósforo y DQO de aguas residuales urbanas de
Maracaibo, Venezuela. Boletín del Centro de Investigaciones Biológicas, 38(2).

Empresa de Acueducto, Alcantarillado y Aseo de Bogotá. (s.f.). Planta de tratamiento de aguas residuales PTAR Salitre. Recuperado de http://www.acueducto.com.co/ guatoc/contingencia/contenidos/ambiental/ptar_ salitre.html

Garbisu, C., Amézaga, I. y Alkorta, I. (2002). Biorremediación y ecología. Ecosistemas, 11(3).

Guerrero, J. (1996). Tratamiento de aguas residuales. Colombia: Universidad Nacional Abierta y a Distancia.

Herrera, N. (2010). Utilización de la microalga Chlorella vulgaris en la remoción de contaminantes orgánicos provenientes de las aguas residuales de la empresa Conalsebos a escala laboratorio (tesis de pregrado). Fundación Universidad de América, Bogotá, Colombia.

Jonte, L., Rosales, N., Yépez, M., Beltrán, B. y Morales, E. (2007). Respuesta de la cianobacteria Synechocystis minuscula a sustratos orgánicos en condiciones mixotróficas. Boletín del Centro de Investigaciones Biológicas, 41(1), 1-136.

Lenntech. (s.f.). Centrifugación y centrifugadores. Recuperado de http://www.lenntech.es/ centrifugacion.htm

Li, Y., Zhou, W., Hu, B., Min, M., Chen, P. y Ruan, R. R. (2012). Effect of light intensity on algal biomass accumulation and biodiesel production for mixotrophic strains Chlorella kessleri and Chlorella protothecoide cultivated in highly concentrated municipal wastewater. Biotechnology and Bioengineering, 109(9), 2222-2229.

Metcalf \& Eddy Inc. (1981). Ingeniería de aguas residuales. Barcelona, España: Editorial Labor, S. A.

Monroy, C. (2014). Cultivo de Chlorella vulgaris en medio bold basal modificado en condiciones de luminosidad baja y caracterización de extracto líquido de lombricomposta como sustituto de medio de cultivo. Xalapa, México: Universidad Veracruzana. 
Moronta, R., Mora, R. y Morales, E. (2006). Respuesta de la microalga Chlorella sorokiniana al $\mathrm{pH}$, salinidad y temperatura en condiciones axenicas y no axenicas. Revista de la Facultad de Agronomía, 23(1).

Muylaert, K., Bastiaens, L., Vandamme, D., \& Gouveia, L. (2018). Harvesting of microalgae: Overview of process options and their strengths and drawbacks. In Microalgae-Based Biofuels and Bioproducts (pp. 113-132).

Olarte, E. y Valencia, M. (2016). Evaluación del uso de la microalga Chlorella vulgaris en el tratamiento de aguas industriales (Vinazas). Colombia: Universidad Nacional Abierta y a Distancia.

Recinto Universitario de Mayagüez. (s.f.). Parámetros fisicoquímicos: alcalinidad. Recuperado de http:// www.uprm.edu/biology/profs/massol/manual/ p2-alcalinidad.pdf

Pearsall, W. y Loose, L. (1936). The growth of Chlorella vulgaris in pure culture. Proceedings of the Royal Society B: Biological Sciences, 121 (824), 451-501.

Ramalho, R. (2003). Tratamiento de aguas residuales. Barcelona, España: Editorial Reverté.

Romero, J. A. (2004). Tratamiento de aguas residuales. Teoría y principios de diseño. Bogotá, Colombia: Escuela Colombiana de Ingeniería.

Tafur, J. E. y Estrada, L. (2015). Tratamiento de aguas residuales in vitro por medio de la microalga Chlorella sp en el municipio de Barrancabermeja, Colombia. Revista CITECSA, 6(10), 5-19.

Universidad del País Vasco. (s.f.). Cámara de contaje Neubauer improved. Recuperado de http://insilico. ehu.es/camara_contaje/neubauer_improved.php

Velasco, L. A., Barros-Gómez, J., Ospina-Salazar, G. H. y Trujillo, C. A. (2009). Efecto de la intensidad lumínica, temperatura y salinidad sobre el crecimiento de la microalga Isochrysis galbana (Clon T-ISO). Intropica, 4, 93-99.

Wang, L., Min, M., Li, Y., Chen, P., Chen, Y., Liu, Y., ... Ruan, R. (2010). Cultivation of green algae Chlorella sp. in different wastewaters from municipal wastewater treatment plant. Applied Biochemistry and Biotechnology, 162(4):1174-1186.

Zhou, W., Li, Y., Min, M., Hu, B., Zhang, H., Ma, X., ... Ruan, R. (2012). Growing wastewater-born microalga Auxenochlorella protothecoides UMN280 on concentrated municipal wastewater for simultaneous nutrient removal and energy feedstock production. Applied Energy, 98, 433-440. 